\title{
Suppression of lysyl-tRNA synthetase, KRS, causes incomplete epithelial-mesenchymal transition and ineffective cell-extracellular matrix adhesion for migration
}

\author{
SEO HEE NAM ${ }^{1,3}$, MINKYUNG KANG ${ }^{2,3}$, JIHYE RYU ${ }^{2,3}$, HYE-JIN KIM ${ }^{2,3}$, DOYEUN KIM ${ }^{2,4}$, \\ DAE GYU KIM ${ }^{2,4}$, NAM HOON KWON ${ }^{2,4}$, SUNGHOON KIM ${ }^{1,2,4}$ and JUNG WEON LEE ${ }^{1-4}$ \\ ${ }^{1}$ Interdisciplinary Program in Genetic Engineering, ${ }^{2}$ Department of Pharmacy, ${ }^{3}$ Tumor Microenvironment \\ Global Core Research Center, ${ }^{4}$ Medicinal Bioconvergence Research Center, Research Institute of Pharmaceutical \\ Sciences, College of Pharmacy, Seoul National University, Seoul 08826, Republic of Korea
}

Received December 16, 2015; Accepted January 20, 2016

DOI: 10.3892/ijo.2016.3381

\begin{abstract}
The cell-adhesion properties of cancer cells can be targeted to block cancer metastasis. Although cytosolic lysyltRNA synthetase (KRS) functions in protein synthesis, KRS on the plasma membrane is involved in cancer metastasis. We hypothesized that KRS is involved in cell adhesionrelated signal transduction for cellular migration. To test this hypothesis, colon cancer cells with modulated KRS protein levels were analyzed for cell-cell contact and cell-substrate adhesion properties and cellular behavior. Although KRS suppression decreased expression of cell-cell adhesion molecules, cells still formed colonies without being scattered, supporting an incomplete epithelial mesenchymal transition. Noteworthy, KRS-suppressed cells still exhibited focal adhesions on laminin, with Tyr397-phopshorylated focal adhesion kinase (FAK), but they lacked laminin-adhesion-mediated extracellular signal-regulated kinase (ERK) and paxillin activation. KRS, p67LR and integrin $\alpha 6 \beta 1$ were found to interact, presumably to activate ERK for paxillin expression and Tyr118 phosphorylation even without involvement of FAK, so that specific inhibition of ERK or KRS in parental HCT116 cells blocked cell-cell adhesion and cell-substrate properties for focal adhesion formation and signaling activity. Together, these results indicate that KRS can promote cell-cell and cellECM adhesion for migration.
\end{abstract}

\section{Introduction}

Cell adhesion involves adhesions comprised of intercellular cell-cell attachments and of cell extracellular matrix protein

Correspondence to: Professor Jung Weon Lee, Department of Pharmacy, College of Pharmacy, Seoul National University, Seoul 08826, Republic of Korea

E-mail: jwl@snu.ac.kr

Key words: anticancer drug, cell-ECM adhesion, epithelialmesenchymal transition, lysyl-tRNA synthetase, migration
(ECM) interactions (1). Proper tuning of the cell adhesion can lead to efficient migration and invasion for a successful metastasis (2). Complicated processes leading to metastasis, migration and invasion should be effectively coordinated by the functions of spatial and temporal cues between cellular and microenvironmental regions (3). Furthermore, regulation of cell adhesion properties may be targeted to control the migration and invasion for cancer metastasis. Diverse signaling or adaptor proteins play critical roles in promoting cancer cells to metastasize (4). Although the signaling molecules contributing to control of the metastatic potentials are very complicated and interconnected, more molecules still need to be identified to understand the control of cellular migration.

Lysyl-tRNA synthetase, KRS, is involved in protein translation. It catalyzes the addition of amino-lysyl to peptides that are being synthesized along the mRNA codons. Although cytosolic KRS is involved in this housekeeping role, KRS at the plasma membranes functions in immune responses $(5,6)$ and tumor metastasis $(7,8)$, clearly indicating non-canonical biological functions besides protein translation. Phosphorylation at the Thr52 residue of KRS by p38 mitogen-activated protein kinase (MAPK) causes dissociation from the cytosolic multi-tRNA synthetase complex (MSC) and translocation to the plasma membrane, where it associates with and stabilizes a $67-\mathrm{kDa}$ laminin receptor (p67LR) for migration and metastasis (7). The p67LR associates with integrin $\alpha 6 \beta 1$ (9) or $\alpha 6 \beta 4$ (10). The integrins are important for cell-extracellular matrix (ECM) adhesion involved in cell migration and invasion (11), as well as signal transduction activation leading to tyrosine phosphorylations of focal adhesion kinase (FAK), paxillin and c-Src involved in cell-ECM adhesion (12). After subcutaneous injection of cells into mice, the interaction between KRS and p67LR can be targeted to inhibit the KRS-dependent metastasis upon a subcutaneous injection of cells to mouse, indicating that KRS can be targeted to block cancer metastasis (8). Although targeting KRS has been shown to inhibit metastasis, how KRS regulates cell-cell adhesion and cell-ECM adhesion is not clearly known.

Cell-cell adhesion involves tight junctions, adherence junctions, gap junctions and desmosomes, whereas cell-ECM 
adhesion occurs at focal adhesions (FAs) and hemidesmosomes (2). Homophilic or heterophilic interactions at the junctions between membrane proteins are important in the epithelial-mesenchymal transition (EMT), and loss of cellcell adhesion and further polarity of the epithelial cells can lead to migratory cells with mesenchymal properties (13). Cell-ECM adhesion is also important in the migration and invasion processes. Acquiring new areas for attachment to the leading edges and detachment from the rear regions must be coordinated for efficient translocation of cell bodies (14). During the coordinated regulation of cell-ECM adhesion, FA dynamics are important (15). During cell adhesion involving integrin to the ECM, cells can activate intracellular signaling molecules such as RhoA GTPases, FAK, c-Src, ERKs and paxillin (16). These activations can lead to the reorganization of F-actin networks to form new integrin-ECM interactions and subsequent tractive force generation (17).

In the present study, the KRS housekeeping protein was characterized for possible regulatory roles in cell-cell adhesion and cell-ECM adhesion related to its pro-migratory functions in HCT116 colon cancer cells. KRS regulated the expression of epithelial markers, including E-cadherin and $\beta$-catenin, involved in cell-cell adhesion. It also regulated cell-laminin adhesion-mediated signaling activities for FAK, ERKs and paxillin. Suppression of KRS resulted in loss of E-cadherin and $\beta$-catenin without a disruption of cell-cell adhesion, and of cell-laminin adhesion-mediated signaling activities, leading to impaired migratory abilities. The results of the present study showed that KRS was able to regulate cell adhesion properties involved in cellular migration, suggesting that it could be a therapeutic target for treatment of colon cancer metastasis.

\section{Materials and methods}

Cells. HCT116 colon cancer cells (American Type Culture Collection, Manassas, VA, USA) were stably transfected with shRNA against KRS, as previously described (18). HCT116 cells overexpressing KRS were established via the stable transfection of a myc-tagged KRS plasmid (7). The cells were maintained in RPMI-1640 medium (Welgene, Daegu, Korea) containing 10\% FBS and antibiotics (Invitrogen, Grand Island, NY, USA).

Extract preparation and western blots. Colon cancer cells were washed with ice-cold phosphate-buffered saline (PBS) and lysed in a modified RIPA buffer (50 mM Tris- $\mathrm{HCl}, 150 \mathrm{mM}$ $\mathrm{NaCl}, 1 \% \mathrm{NP}-40$ and $0.25 \%$ sodium deoxycholate) with a protease inhibitor cocktail (GenDepot, Barker, TX, USA). The extracts were centrifuged for $30 \mathrm{~min}$ at $15,000 \mathrm{xg}$ at $4^{\circ} \mathrm{C}$, and then the lysates were collected from the supernatant. The protein amounts were normalized, before further immunoblotting with different antibodies. The primary antibodies used in the study were as follows and were against: $\alpha$-tubulin (Sigma-Aldrich, St. Louis, MO, USA); $\mathrm{pY}^{416} \mathrm{c}-\mathrm{Src}$, ERKs, and phospho-ERKs (Cell Signaling Technology, Danvers, MA, USA); paxillin and FAK (BD Biosciences, San Jose, CA, USA); pY ${ }^{397}$ FAK (Abcam, Cambridge, UK); c-Src, pY ${ }^{118}$ paxillin, $\mathrm{pY}^{577} \mathrm{FAK}, \mathrm{pY}^{861} \mathrm{FAK}, \mathrm{pY}^{925} \mathrm{FAK}$, Snaill, E-cadherin and $\beta$-catenin (Santa Cruz Biotechnology, Santa Cruz, CA, USA), integrin $\alpha 6$ and $\beta 1$ (Millipore, Billerica, MA, USA); and KRS (Atlas Antibodies, Stockholm, Sweden).

Normal culture or replating on ECM layer. Cells were kept in suspension or replated on serum (10\%) or ECM $(10 \mu \mathrm{g} / \mathrm{ml}$ laminin; BD Biosciences) -precoated dishes or coverglasses in the presence of $2 \%$ FBS for $1 \mathrm{~h}$, before being analyzed for cell-serum or -laminin adhesion signaling by standard western blotting, as previously described (19). Pharmacological inhibitors were added to the culture media for $24 \mathrm{~h}$ or to replating media at the reseeding time. Cells were harvested for whole cell lysates, prior to immunoblottings for the indicated proteins, or imaging to monitor cell growth patterns. Confluent cells were wounded with a pipette tip and washed twice with PBS. After treatment with DMSO, U0126 $(10 \mu \mathrm{M})$ LC Laboratories, Woburn, MA, USA), or YH16899 $(10 \mu \mathrm{M})$ and incubation in a $\mathrm{CO}_{2}$ incubator for $24 \mathrm{~h}$, the marginal edges of the wounds were imaged (CKX41; Olympus, Tokyo, Japan).

Coimmunoprecipitations. HCT116 cells stably expressing myc-KRS WT in standard media containing $10 \%$ FBS in the presence of control vehicle or YH16899 treatment $(10 \mu \mathrm{M})$ for $24 \mathrm{~h}$ were harvested for whole cell lysates, and lysates were immunoprecipitated overnight using anti-myc tagged antibody-coated agarose beads (Sigma-Aldrich). The immunoprecipitated proteins were boiled in 2X SDS-PAGE sample buffer before standard western blot analyses.

Indirect immunofluorescence. Cells reseeded in normal culture media- or laminin-precoated glass coverslips in $2 \%$ FBS-containing media for 2 or $24 \mathrm{~h}$ were stained for F-actin using phalloidin, or immunostained using antibodies against E-cadherin, $\beta$-catenin, N-cadherin, pERK, phospho-Tyr397, or phospho-Tyr118 paxillin, in addition to 4',6-diamidino-2-phenylindole (DAPI) staining for the nucleus. Immunofluorescence images were acquired on a fluorescence microscope (BX51TR; Olympus) or on a confocal laser scanning microscope with a Nikon Plan Apochromat 60x/1.4 N.A. oil objective (Nikon Eclipse Ti microscope; Nikon, Tokyo, Japan).

$R T-P C R$. Total RNA was extracted from cells under diverse experimental conditions, using TRIzol ${ }^{\circledR}$ (Invitrogen) according to the manufacturer's protocol. Total RNA $(1 \mu \mathrm{g})$ was reverse transcribed using the amfiRivert Platinum cDNA Synthesis Master Mix (GenDepot). Primers were designed using Primer3 software as follows: human E-cadherin $(\mathrm{CDH}) \mathrm{mRNA}$, forward 5'-TGCCCAGAAAATGAAAAAGG-3' and reverse 5'-GTGTATGTGGCAATGCGTTC-3'; human N-cadherin $(\mathrm{CDH} 2)$ mRNA, forward 5'-ACAGTGGCCACCTACAAA GG-3' and reverse 5'-CCGAGATGGGGTTGATAATG-3'; human vimentin (VIM) mRNA, forward 5'-GAGAACTTTG CCGTTGAAGC-3' and reverse 5'-GCTTCCTGTAGGTGGC AATC-3'; human Twist (TWIST) mRNA, forward 5'-GGAGT CCGCAGTCTTACGAG-3' and reverse 5'-TCTGGAGGACC TGGTAGAGG-3'; human paxillin $(P X N)$ mRNA, forward 5'-GAAATCAGCTGAGCCTTCAC-3' and reverse 5'-TTAG GCTTCTCTTTCGTCAGG-3', Snaill, forward 5'-GGTTC TTCTGCGCTACTGCT-3' and reverse 5'-TAGGGCTGCTG GAAGGTAAA-3'; slug, forward 5'-GGGGAGAAGCCTTT TTCTTG-3' and reverse 5'-TCTCATGTTTGTGCAGGAG-3'; 

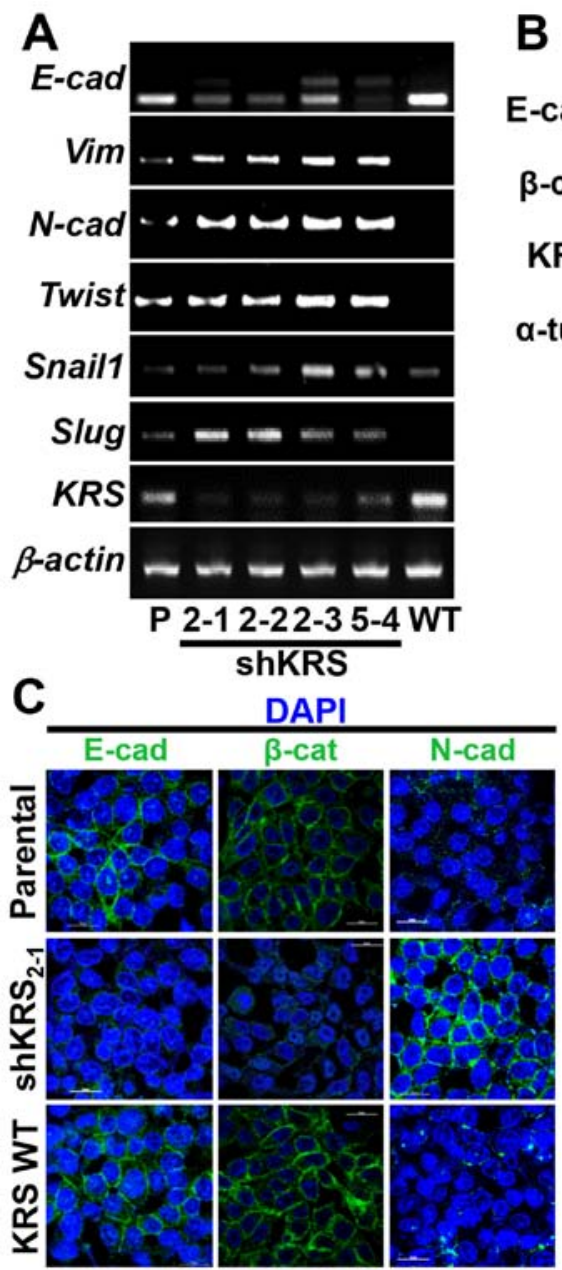

B

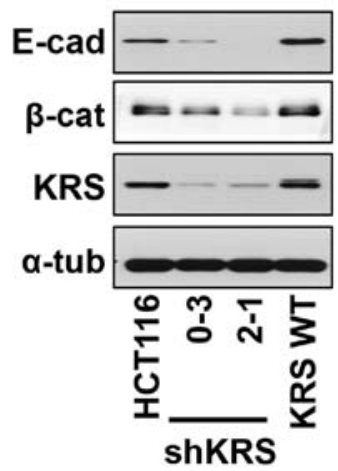

D
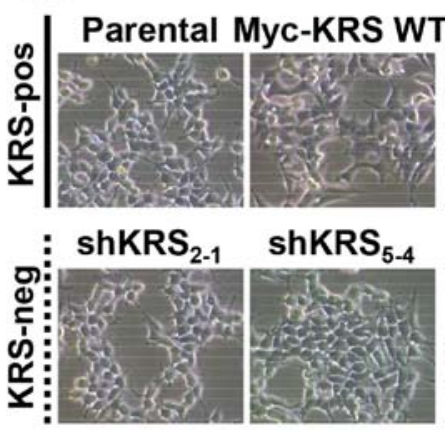

E

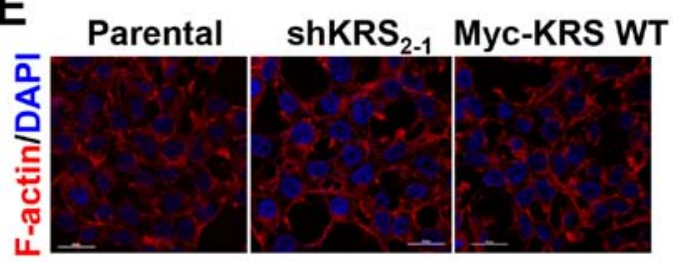

$\mathbf{F}$

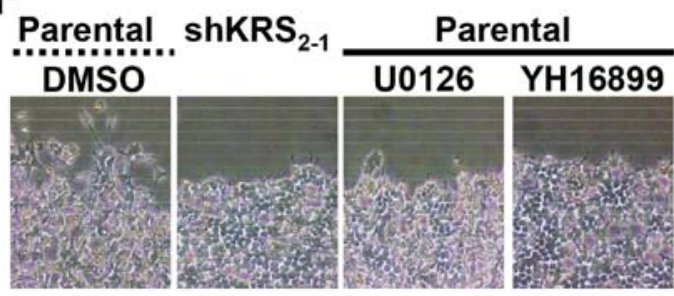

Figure 1. KRS downregulation causes incomplete EMT phenotypes. (A and B) RT-PCR (A) or standard western blots (B) for the indicated epithelial or mesenchymal markers were performed using KRS-expressing parental HCT116 (P), stably myc-KRS wild-type overexpressing HCT116 (KRS WT), and stably KRS-suppressed HCT116 cell clones (2-1, 2-2, 2-3 and 5-4 for shKRS) normally cultured in 10\% FBS-containing condition. (C) Cells were cultured on coverglasses precoated with $10 \%$ FBS-containing culture media until confluence and then immunostained for the molecules along with DAPI costaining. (D and E) Subconfluent cells from the normal culture condition were imaged using a phase contrast microscope (D) or stained for F-actin before visualization using a fluorescent microscope (E). (F) Confluent cells grown under normal conditions were wounded, washed and treated with either DMSO or the indicated inhibitor. Fifteen hours later, cells near the wound edges were randomly imaged. Data shown represent at least three independent experiments.

human KRS (KARS) mRNA, forward 5'-CAATGCCCATGC CCCAGCCA-3' and reverse 5'-ACCCCACCCTTCCGGCG AAT-3'; human $\beta$-actin $(A C T B)$ mRNA, forward 5'-TGAC GGGGTCACCCACACTGTGCCCATCTA-3' and reverse 5'-CTAGAAGCATTTGCGGTGGACGACGGAGGG-3'.

Statistical methods. The Student's t-test was performed for statistical comparisons of mean values to determine significance. A P-value $<0.05$ was considered to indicate a statistically significant result.

\section{Results}

KRS suppression caused an incomplete EMT phenotype. To explore the roles of KRS in metastatic potential, HCT116 colon cancer cells with endogenous, suppressed, or overexpressed KRS levels were first analyzed for EMT phenotypes. Using an RT-PCR approach, we found that KRS suppression decreased E-cadherin mRNA but increased the mRNAs of mesenchymal markers (Fig. 1A). Furthermore, these KRS suppression-dependent changes in EMT markers also applied to protein levels; epithelial markers, including E-cadherin, were expressed at higher levels in KRS-expressing parental and KRS-overexpressing HCT116 cells, whereas KRS-suppressed cell clones had decreased E-cadherin and $\beta$-catenin expression (Fig. 1B). We then checked whether KRS downregulation could affect general protein translation and/or apoptosis, and there were no differences between KRS-expressing and KRS-suppressed cell clones (18). E-cadherin was not clearly observed in cell-cell junctions but $\mathrm{N}$-cadherin was expressed in the junctions with minimal $\beta$-catenin when KRS was suppressed, as compared with KRS-expressing cells (Fig. 1C). Although the inverse relationship between KRS and epithelial marker expression might suggest the induction of EMT phenotypes, cell-cell contacts were not disrupted, presumably due to N-cadherin at the junctions after KRS suppression (Fig. 1C and D). Furthermore, cortical actin, but not stress fibers, was observed at the cell junctions independent of KRS expression (Fig. 1E). These observations may suggest that KRS suppression caused a partial EMT phenotype.

However, when wounds were made in confluent cells in normal $10 \%$ fetal bovine serum (FBS)-containing conditions, 
A
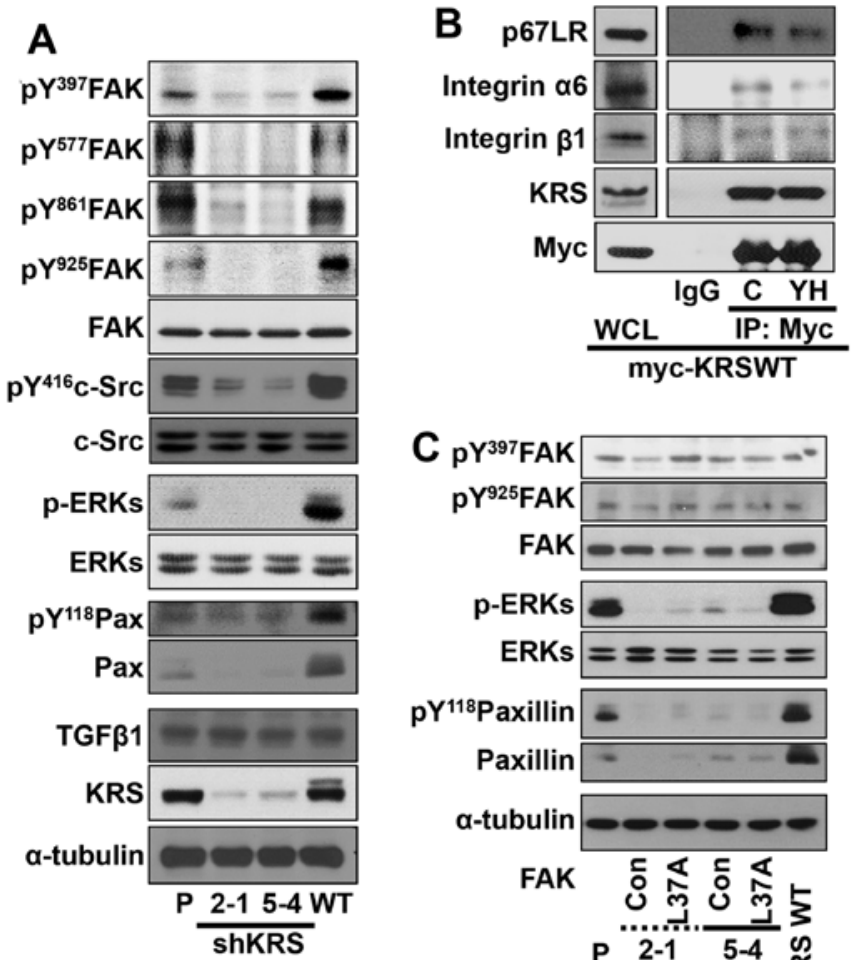

myc-KRSWT

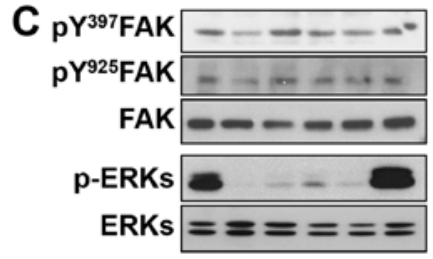

pY ${ }^{118}$ Paxillin

Paxillin - $-w$

a-tubulin $-\infty$

FAK
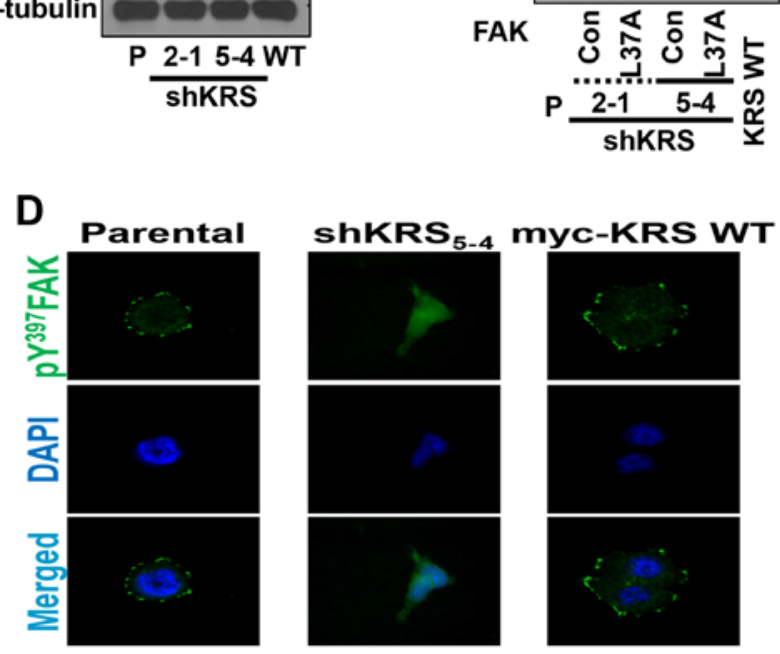

Figure 2. KRS suppression impairs cell adhesion-related signaling activities (A) HCT116 cells with various KRS expression levels [parental P, KRSsuppressed 2-1 and 5-4 clones, and myc-KRS WT overexpressed (WT) cells] were analyzed for the indicated molecules via standard western blots. (B) Myc-KRS WT cells grown in normal culture conditions in the presence of vehicle DMSO (C) or YH16899 (YH) treatment were harvested for whole cell lysates (WCL) prior to immunoprecipitation using normal $\operatorname{IgG}(\operatorname{IgG})$ or anti-myc antibody and standard western blotting for the indicated molecules. (C) Stable HCT116 cells were transfected with control vector (Con) or L37A FAK mutant (L37A) for $48 \mathrm{~h}$, before whole cell lysates preparation and standard western blots for the indicated molecules. (D) Cells replated overnight on coverglasses precoated with $10 \%$ FBS containing normal culture media were stained with DAPI for nuclei and immunostained for phospho-Tyr397 $\left(\mathrm{pY}^{397} \mathrm{FAK}\right)$, prior to saving the representative images using a fluorescent microscopy. Data shown represent three independent experiments.

the KRS-expressing parental cells exhibited clear outbound movement at the wound boundary, whereas KRS-suppressed cells did not (Fig. 1F). These KRS-dependent differential movements toward free spaces might be correlated with ineffective cell-substrate adhesion processes.

KRS suppression impaired cellular signaling and focal adhesion formation. We then examined the relationship between KRS expression and cell-ECM adhesion properties. First,

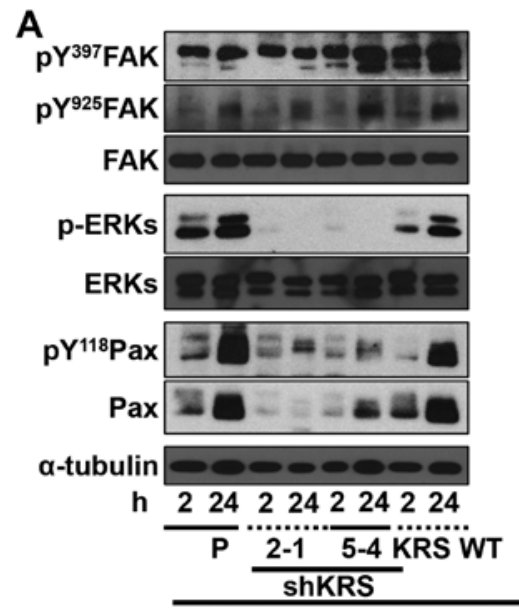

HCT116 on 2D laminin w/ 2\% FBS

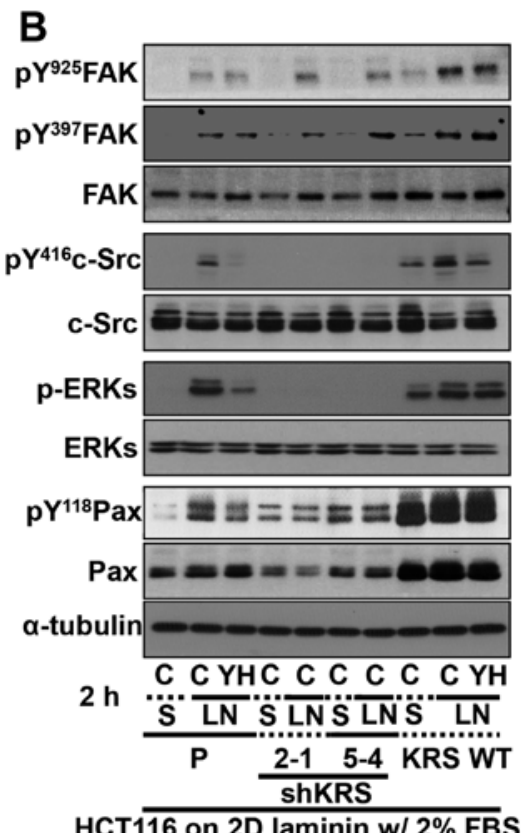

Figure 3. KRS suppression impairs signaling activities mediated by cell-laminin adhesion. (A) Cells were reseeded onto laminin (LN)-precoated dishes under $2 \%$ FBS containing culture media for 2 or $24 \mathrm{~h}$, prior to harvesting for standard western blots for the indicated molecules. (B) Cells were kept in suspension (S) or reseeded onto laminin (LN)-precoated dishes under $2 \%$ FBS containing culture media with DMSO (C) or YH16899 (YH) treatment for $2 \mathrm{~h}$, prior to harvesting for standard western blots for the indicated molecules. Data shown represent three independent experiments.

cells with various KRS expression levels were allowed to adhere under normal 10\% FBS-containing culture conditions and were examined for adhesion-related signaling activities. Under normal 10\% FBS-containing conditions, KRS suppression abolished the signaling activities of FAK, ERK, c-Src and paxillin, and the expression of paxillin, compared with KRS-expressing (or -overexpressing) cells (Fig. 2A). Integrins activate FAK, ERK and paxillin for diverse cellular functions in different cell systems (16). KRS is translocated to the plasma membrane when it associates and collaborates with p67LR (a non-integrin laminin receptor) in the presence of extracellular laminin, following Thr52-phosphorylation-dependent disassociation from cytosolic MSC (7). Integrin $\alpha 6 \beta 1$ and $\alpha 6 \beta 4$ respond to laminin and bind p67LR $(9,10)$. Therefore, to 

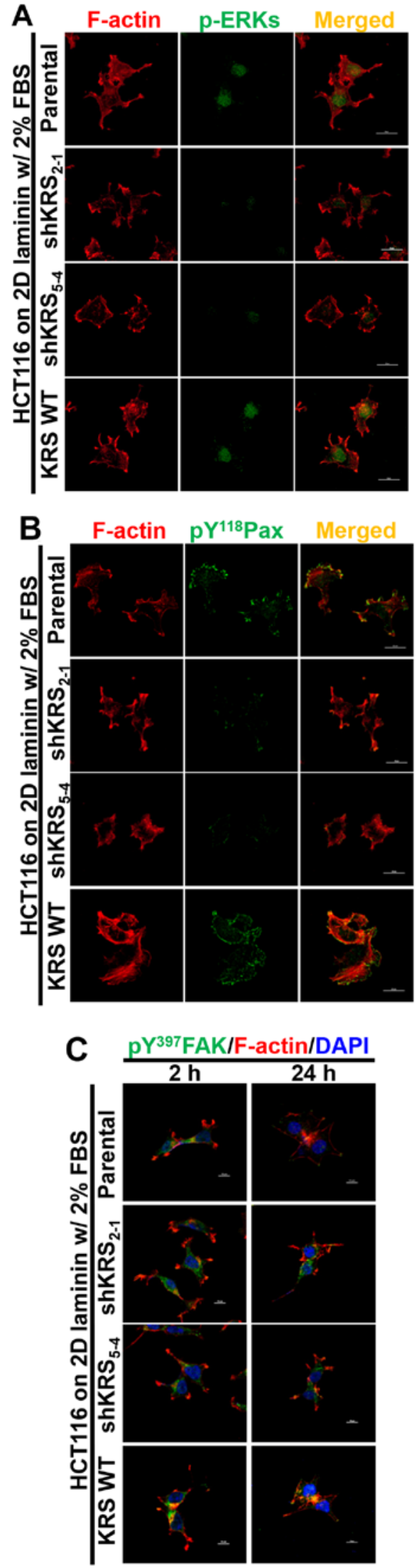

Figure 4. KRS suppression impairs formation of FAs with phospho-Tyr118 paxillin upon adhesion onto laminin. (A) Cells were reseeded onto laminin (LN)-precoated coverglasses under $2 \%$ FBS containing culture media for 2 (A-C) or $24 \mathrm{~h}(\mathrm{C})$, prior to staining for F-actin and immunostaining for pERKs (A), phospho-Tyr118 paxillin (pY ${ }^{118}$ Paxillin, B), and phospho-Tyr397 FAK ( $\left.\mathrm{pY}^{397} \mathrm{FAK}, \mathrm{C}\right)$. The representative images for each condition were selected among more than 10 random images and shown. Data shown represent three independent experiments. determine whether the interaction between KRS, p67LR and integrins could be correlated to signaling activation for FAK, c-Src, ERKs and paxillin, we examined possible physical interactions between these proteins via a coimmunoprecipitation study. Myc-KRS in extracts prepared from normal $10 \%$ FBS-containing culture conditions co-precipitated integrins $\alpha 6$, and $\beta 1$ and this interaction was blocked by the anti-KRS inhibitor (YH16899) treatment (Fig. 4C) (8).

In addition, stable transfection of L37A mutant FAK, which was shown to be active (20), into KRS-suppressed cells did not cause ERK activation, paxillin expression and Tyr118 phosphorylation (Fig. 2C), suggesting that KRS might regulate ERKs/paxillin signaling activity and expression independently of FAK activation. Furthermore, when phospho-Tyr397 FAK was imaged to visualize the focal adhesions (FAs), KRS-suppressed cells did not show FAs, whereas KRS-expressing cells showed well-formed FAs (Fig. 2D).

Cell adhesion-related signaling activities were then analyzed after the cells were kept in suspension or reseeded onto ECM-precoated culture dishes. Because KRS has been shown to translocate to the plasma membrane in a laminin-dependent manner (7), and because KRS suppression did not change laminin expression (18), we explored the biological significance of KRS expression after reseeding onto laminin-precoated plates under reduced (2\%) serumcontaining conditions. FAK phosphorylation depended on KRS expression in cases of parental or overexpressing cells, as reported previously showing that KRS overexpression in A549 cells further increased Tyr397 FAK phosphorylation upon being replated onto laminin-precoated dishes in the absence of serum (7). However, FAK phosphorylations in KRS-suppressed cells were somewhat comparable to that in parental HCT116 cells (Fig. 3A). KRS-dependent FAK activity in $10 \%$ serum-containing normal conditions (Fig. 2A) appeared different from HCT116 cells manipulated and newly adhered onto laminin under a lower serum conditions (Fig. 3A). The adhesion-dependent phosphorylation of FAK in KRS-expressing cells under these conditions was not affected by treatment with a KRS inhibitor YH16899 (Fig. 3B), which blocks the interaction between KRS and p67LR (Fig. 2B). However, the phosphorylation of ERK, paxillin and c-Src in KRS-expressing cells increased upon adhesion to laminin and was abolished by additional YH16899 treatment, whereas no effects were observed in KRS-suppressed cells (Fig. 3B).

We next examined the subcellular localization of phosphoERK, phospho-Tyr118 paxillin, or phospho-Tyr397 FAK in cells with various KRS expression levels. When the cells were reseeded onto laminin-precoated coverglasses in $2 \%$ serumcontaining media, KRS-positive (but not KRS-suppressed) cells showed obvious phospho-ERK staining in the nucleus (Fig. 4A). Phospho-Tyr118 paxillin was also clearly localized to FAs in a KRS-dependent manner (Fig. 4B). Notably, replating the cells onto laminin for 2 or $24 \mathrm{~h}$ resulted in comparable protrusions and spreading with comparable phospho-Tyr397 FAK levels, independent of KRS suppression (Fig. 4C). This suggested that FAK activity in response to laminin stimulation might not depend on KRS expression. However, ERK1/2 phosphorylation and paxillin expression and phosphorylation depended on KRS expression levels (Figs. 3 and 4). 

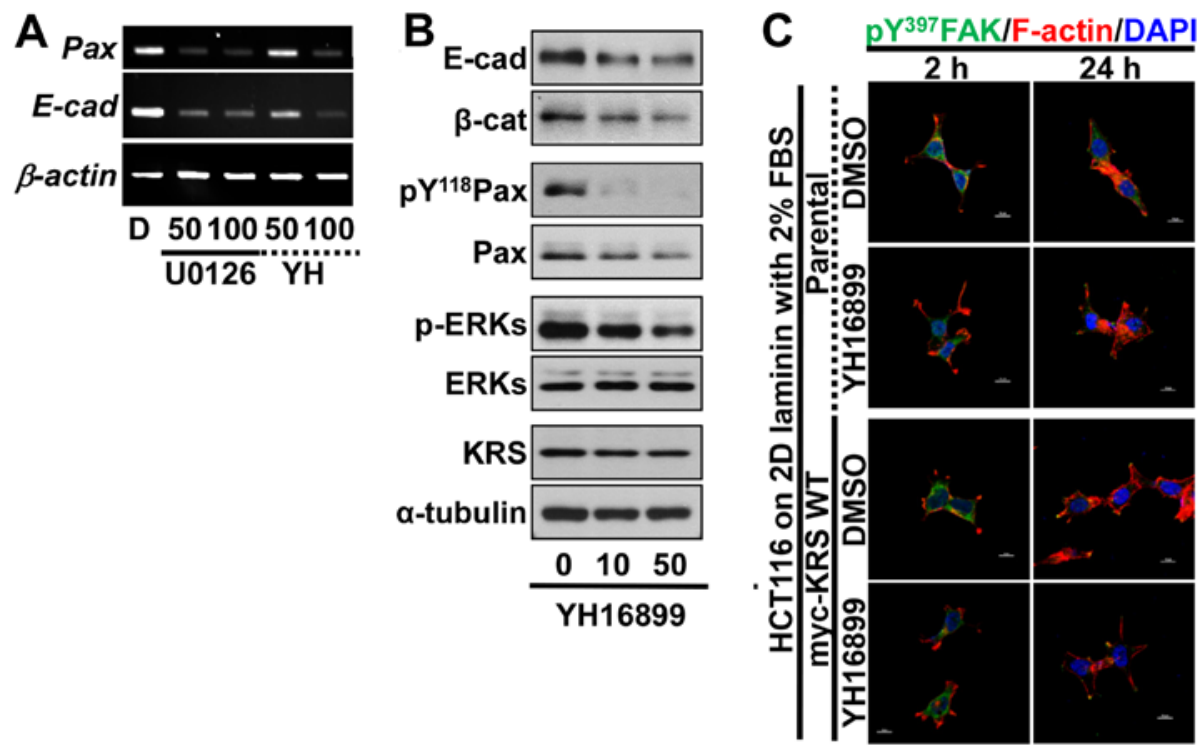

D HCT116 on $2 D$ laminin $w / 2 \%$ FBS

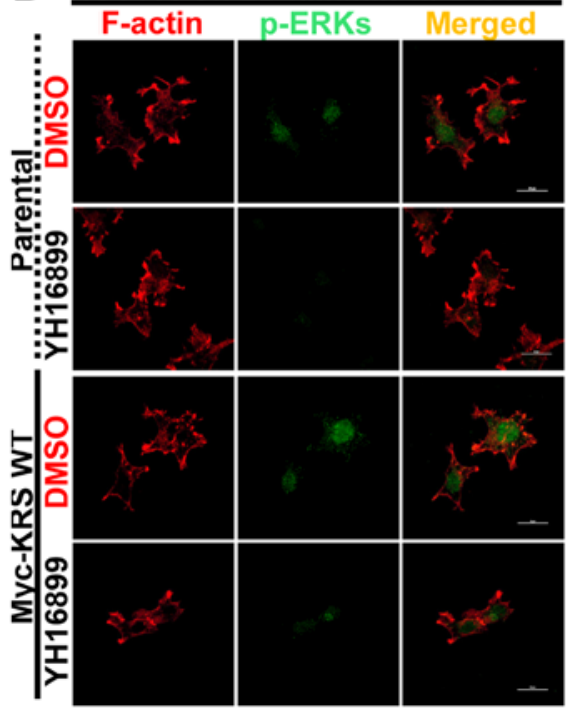

E HCT116 on $2 \mathrm{D}$ laminin $\mathrm{w} / 2 \% \mathrm{FBS}$

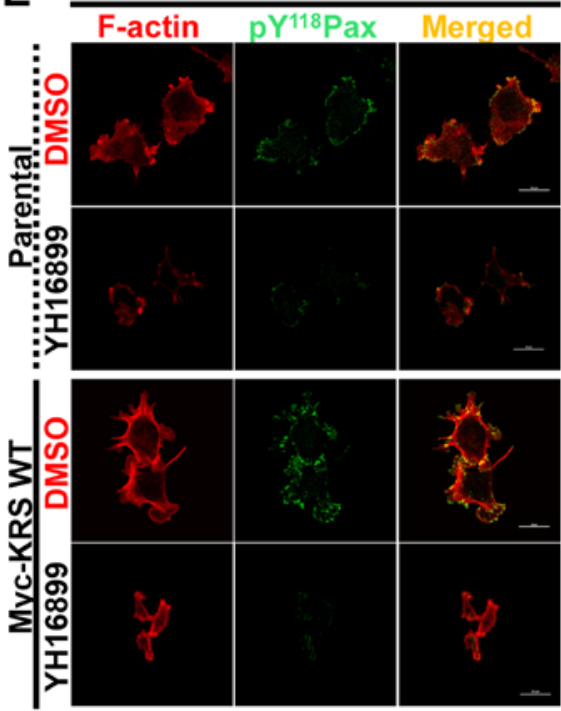

Figure 5. Anti-KRS reagent impairs formation of FAs with phospho-Tyr118 paxillin on cell-laminin adhesion. (A and B) HCT116 parental cells were treated with U0126 (50 or $100 \mu \mathrm{M})$ or YH16899 $(50$ or $100 \mu \mathrm{M})$ for $24 \mathrm{~h}$, and the cells were then processed for RT-PCR (A) or immunoblotting (B) for the indicated molecules. (C-E) Cells were reseeded onto laminin-precoated coverglasses for $2 \mathrm{~h}$ in the presence of DMSO (vehicle) or YH16899 (50 mM) treatment before immunostaining for $\mathrm{pY}^{397} \mathrm{FAK}\left(\mathrm{C}\right.$, green), $\mathrm{pERK}$ (D, green), or $\mathrm{pY}^{118}$ Paxillin (E, green) with phalloidin for F-actin (red) and DAPI for DNA (blue) costaining. Data represent three independent experiments.

Anti-KRS reagent abolishes $K R S$-dependent cell adhesion properties. We then explored whether KRS functioned on cell adhesion properties, using the anti-KRS reagent YH16899. Treating KRS-expressing cells with either U0126 or YH16899 decreased both paxillin and E-cadherin mRNA levels (Fig. 5A). Upon KRS inhibition by the YH16899 treatment, E-cadherin and $\beta$-catenin proteins also decreased, similarly to ERK and paxillin phosphorylations (Fig. 5B). However, YH16899 treatment of KRS-expressing cells did not result in changes in protrusion and spreading as visualized by Tyr397-phosphorylated FAK staining (Fig. 5C), but did result in decreased phospho-ERK (Fig. 5D) and phospho-Tyr118 paxillin (Fig. 5E), under the laminin-precoated conditions containing 2\% FBS. These observations, therefore, suggested that the KRS-dependent cell adhesion activity could involve ERK and paxillin expression and activity.

\section{Discussion}

The present study suggests that KRS functions regulating cell-cell adhesion properties, leading to incomplete EMT, and cell-laminin adhesion-dependent signaling via an association with p67LR and integrin $\alpha 6 \beta 1$ receptors. The roles of KRS in regulating cell-cell intercellular and cell-substrate adhesions by regulating E-cadherin expression, ERK activity, and paxillin expression and activity could promote cellular migration. During the KRS-dependent activation of the cell-substrate adhesion signaling pathway, FAK appeared unrelated to ERKs, paxillin activation and paxillin expression. This KRS/ERK/paxillin signaling axis may be a useful target against KRS-dependent cancer metastases.

KRS suppression modulated the epithelial-mesenchymal properties of cells; KRS suppression decreased the expression 
of epithelial markers and concomitantly increased mesenchymal markers. However, how KRS causes these alterations should be explored further. Because mesenchymal markers increased upon KRS suppression, a possible global effect on protein translational capacity from the downregulated level of KRS was not the cause. Furthermore, KRS suppression did not cause cellular loss of adherence even after E-cadherin loss, presumably because mesenchymal $\mathrm{N}$-cadherin replaced epithelial E-cadherin at the adherence junctions of KRS-suppressed cells. In addition to its roles in cell-cell contacts, KRS promoted cell-substrate adhesion signaling, which is important for myosin contractility-dependent adhesion strength and traction force (21) and for cellular contractility (22). Thus, KRS-mediated migration is involved in cell-substrate adhesion for contractility/traction force, so that cells can 'crawl out', even in the cases where cell-cell adhesion is well-formed via adherence junctions. The early phases of colon cancer metastasis are suggested to have adapted EMT-like de-differentiation at the invasive edges, in order to detach and migrate (23). HCT116 cells quite highly express E-cadherin and are categorized as the most epithelial-like cells in the EMT score among the diverse tumor cell types (24). Presumably, E-cadherin for cellcell adhesions and cell-substrate adhesion in KRS-positive colon cancer cells may coordinately contribute to migration for efficient metastasis involving a transient EMT-like process. We observed that KRS-dependent migration did not depend solely on either epithelial or mesenchymal characteristics, because KRS-suppressed cells could not efficiently migrate, although they obviously lost E-cadherin and other epithelial markers. It was recently reported that circulating tumor cells (CTCs) originated from primary breast tumors consisting of cells with epithelial, mesenchymal, or both characteristics $(25,26)$. Therefore, it is likely that KRS-expressing cells with certain epithelial characteristics have efficient migration.

KRS-suppressed cells did not move away from the boundaries of cell masses, therefore, the loss of epithelial markers was not sufficient for the outward movement. In addition to regulating cell-cell adhesion, KRS could also regulate cellsubstrate adhesion as part of its pro-metastatic functions. KRS translocates from the cytosolic MSC to the plasma membrane after p38 MAPK-dependent Thr52 phosphorylation upon extracellular laminin stimulation, where it protects p67LR from ubiquitination-mediated degradation (7). Furthermore, the interaction between KRS and p67LR is critically involved in the lung metastasis of subcutaneously-injected mouse breast carcinoma 4T1 cells with KRS overexpression (8). The KRS/p67LR complex also included integrin $\alpha 6 \beta 1$, allowing KRS-expressing cells to transduce intracellular signals under extracellular laminin-stimulated conditions. Moreover, extracellular laminin, but not collagen I, causes KRS translocation to the plasma membrane for binding to p67LR (7). Thus, it is likely that KRS may mediate ERK activation for prometastatic roles following specific adhesion-mediated signals from the extracellular microenvironment.

In the present study, KRS-dependent migration was shown to require ERK activity and paxillin expression and activity, leading to efficient FA formation; inactivating ERK or disrupting complex formation among KRS, p67LR and integrin $\alpha 6 \beta 1$ blocked KRS-dependent effects. FAK, c-Src family kinases (SFKs), paxillin, and ERK1/2 are key regulators of focal adhesion dynamics, especially during cell adhesion and migration $(27,28)$. Cell-substrate adhesion activates ERK via a FAK Tyr925 phosphorylation-mediated signaling pathway from FAK to the Ras cascade upon integrin/ECM interaction (29). However, FAK activity was not relevant for the KRS-dependent ERK and paxillin activations and paxillin expression, because manipulation of FAK activity did not lead to ERK activity and paxillin expression/Tyr118 phosphorylation. FAK-independent ERK activation may occur during cell adhesion events. The association of integrins with caveolin-1 and Fyn (a SFK member) recruits Shc for ERK activation (30-32). Furthermore, the cell adhesion-dependent activation of CaMKII in vascular smooth muscle cells leads to ERK activation independent of FAK activity (33).

The signaling transduced from the KRS/p67LR/integrin $\alpha 6 \beta 1$ complex to paxillin expression/phosphorylation via ERK activity could be targeted to treat colon cancer metastasis depending on KRS expression and functions.

\section{Acknowledgements}

The present study was supported by the National Research Foundation of Korea (NRF) grant for the Tumor Microenvironment Global Core Research Center (GCRC) funded by the Korea government (Ministry of Science, ICT \& Future Planning) (2011-0030001 to J.W.L.), for the Senior Researchers Program (Leap research, 2012-0005606/2013035235 to J.W.L.), and for the Medicinal Bioconvergence Research Center (NRF-M1AXA002-2010-0029785 to S.K. and NRF-2012M3A6A4054271 to J.W.L.).

\section{References}

1. Ridley AJ, Schwartz MA, Burridge K, Firtel RA, Ginsberg MH, Borisy G, Parsons JT and Horwitz AR: Cell migration: Integrating signals from front to back. Science 302: 1704-1709, 2003.

2. Collins $\mathrm{C}$ and Nelson WJ: Running with neighbors: Coordinating cell migration and cell-cell adhesion. Curr Opin Cell Biol 36: 62-70, 2015.

3. Geiger B, Spatz JP and Bershadsky AD: Environmental sensing through focal adhesions. Nat Rev Mol Cell Biol 10: 21-33, 2009.

4. Geiger T and Geiger B: Towards elucidation of functional molecular signatures of the adhesive-migratory phenotype of malignant cells. Semin Cancer Biol 20: 146-152, 2010.

5. Park SG, Kim HJ, Min YH, Choi EC, Shin YK, Park BJ, Lee SW and Kim S: Human lysyl-tRNA synthetase is secreted to trigger proinflammatory response. Proc Natl Acad Sci USA 102: 6356-6361, 2005

6. Yannay-Cohen N, Carmi-Levy I, Kay G, Yang CM, Han JM, Kemeny DM, Kim S, Nechushtan H and Razin E: LysRS serves as a key signaling molecule in the immune response by regulating gene expression. Mol Cell 34: 603-611, 2009.

7. Kim DG, Choi JW, Lee JY, Kim H, Oh YS, Lee JW, Tak YK, Song JM, Razin E, Yun SH, et al: Interaction of two translational components, lysyl-tRNA synthetase and p40/37LRP, in plasma membrane promotes laminin-dependent cell migration. FASEB J 26: 4142-4159, 2012.

8. Kim DG, Lee JY, Kwon NH, Fang P, Zhang Q, Wang J Young NL, Guo M, Cho HY, Mushtaq AU, et al: Chemical inhibition of prometastatic lysyl-tRNA synthetase-laminin receptor interaction. Nat Chem Biol 10: 29-34, 2014.

9. Canfield SM and Khakoo AY: The nonintegrin laminin binding protein (p67 LBP) is expressed on a subset of activated human $\mathrm{T}$ lymphocytes and, together with the integrin very late activation antigen-6, mediates avid cellular adherence to laminin. J Immunol 163: 3430-3440, 1999. 
10. Ardini E, Tagliabue E, Magnifico A, Butò S, Castronovo V, Colnaghi MI and Ménard S: Co-regulation and physical association of the $67-\mathrm{kDa}$ monomeric laminin receptor and the alpha6beta4 integrin. J Biol Chem 272: 2342-2345, 1997.

11. Berno V, Porrini D, Castiglioni F, Campiglio M, Casalini P, Pupa SM, Balsari A, Ménard S and Tagliabue E: The $67 \mathrm{kDa}$ laminin receptor increases tumor aggressiveness by remodeling laminin-1. Endocr Relat Cancer 12: 393-406, 2005.

12. Carragher NO and Frame MC: Focal adhesion and actin dynamics: A place where kinases and proteases meet to promote invasion. Trends Cell Biol 14: 241-249, 2004.

13. Lamouille S, Xu J and Derynck R: Molecular mechanisms of epithelial-mesenchymal transition. Nat Rev Mol Cell Biol 15: 178-196, 2014

14. Scales TM and Parsons M: Spatial and temporal regulation of integrin signalling during cell migration. Curr Opin Cell Biol 23: 562-568, 2011.

15. Albiges-Rizo C, Destaing O, Fourcade B, Planus E and Block MR: Actin machinery and mechanosensitivity in invadopodia, podosomes and focal adhesions. J Cell Sci 122: 3037-3049, 2009.

16. Lee JW and Juliano R: Mitogenic signal transduction by integrin-and growth factor receptor-mediated pathways. Mol Cells 17: 188-202, 2004

17. Valdembri D and Serini G: Regulation of adhesion site dynamics by integrin traffic. Curr Opin Cell Biol 24: 582-591, 2012.

18. Nam SH, Kim D, Lee MS, Lee D, Kwak TK, Kang M, Ryu J, Kim HJ, Song HE, Choi J, et al: Noncanonical roles of membranous lysyl-tRNA synthetase in transducing cell-substrate signaling for invasive dissemination of colon cancer spheroids in 3D collagen I gels. Oncotarget 6: 21655-21674, 2015.

19. Lee SA, Kim YM, Kwak TK, Kim HJ, Kim S, Ko W, Kim SH, Park KH, Kim HJ, Cho M, et al: The extracellular loop 2 of TM4SF5 inhibits integrin alpha2 on hepatocytes under collagen type I environment. Carcinogenesis 30: 1872-1879, 2009.

20. Jung O, Choi S, Jang SB, Lee SA, Lim ST, Choi YJ, Kim HJ, Kim DH, Kwak TK, Kim H, et al: Tetraspan TM4SF5-dependent direct activation of FAK and metastatic potential of hepatocarcinoma cells. J Cell Sci 125: 5960-5973, 2012.

21. Dumbauld DW, Lee TT, Singh A, Scrimgeour J, Gersbach CA, Zamir EA, Fu J, Chen CS, Curtis JE, Craig SW, et al: How vinculin regulates force transmission. Proc Natl Acad Sci USA 110: 9788-9793, 2013

22. Clark K, Howe JD, Pullar CE, Green JA, Artym VV, Yamada KM and Critchley DR: Tensin 2 modulates cell contractility in 3D collagen gels through the RhoGAP DLC1. J Cell Biochem 109: 808-817, 2010
23. Chua KN, Poon KL, Lim J, Sim WJ, Huang RY and Thiery JP: Target cell movement in tumor and cardiovascular diseases based on the epithelial-mesenchymal transition concept. Adv Drug Deliv Rev 63: 558-567, 2011.

24. Huang RY, Wong MK, Tan TZ, Kuay KT, Ng AH, Chung VY, Chu YS, Matsumura N, Lai HC, Lee YF, et al: An EMT spectrum defines an anoikis-resistant and spheroidogenic intermediate mesenchymal state that is sensitive to e-cadherin restoration by a src-kinase inhibitor, saracatinib (AZD0530). Cell Death Dis 4: e915, 2013.

25. Thiery JP and Lim CT: Tumor dissemination: An EMT affair. Cancer Cell 23: 272-273, 2013.

26. Yu M, Bardia A, Wittner BS, Stott SL, Smas ME, Ting DT, Isakoff SJ, Ciciliano JC, Wells MN, Shah AM, et al: Circulating breast tumor cells exhibit dynamic changes in epithelial and mesenchymal composition. Science 339: 580-584, 2013.

27. Panetti TS: Tyrosine phosphorylation of paxillin, FAK, and p130CAS: Effects on cell spreading and migration. Front Biosci 7: d143-d150, 2002.

28. Webb DJ, Donais K, Whitmore LA, Thomas SM, Turner CE, Parsons JT and Horwitz AF: FAK-Src signalling through paxillin, ERK and MLCK regulates adhesion disassembly. Nat Cell Biol 6: 154-161, 2004.

29. Schlaepfer DD, Hanks SK, Hunter T and van der Geer P: Integrin-mediated signal transduction linked to Ras pathway by GRB2 binding to focal adhesion kinase. Nature 372: 786-791, 1994.

30. Lin TH, Aplin AE, Shen Y, Chen Q, Schaller M, Romer L, Aukhil I and Juliano RL: Integrin-mediated activation of MAP kinase is independent of FAK: Evidence for dual integrin signaling pathways in fibroblasts. J Cell Biol 136: 1385-1395, 1997.

31. Wary KK, Mainiero F, Isakoff SJ, Marcantonio EE and Giancotti FG: The adaptor protein Shc couples a class of integrins to the control of cell cycle progression. Cell 87: 733-743, 1996.

32. Wary KK, Mariotti A, Zurzolo C and Giancotti FG: A requirement for caveolin-1 and associated kinase Fyn in integrin signaling and anchorage-dependent cell growth. Cell 94: 625-634, 1998

33. Lu KK, Armstrong SE, Ginnan R and Singer HA: Adhesiondependent activation of CaMKII and regulation of ERK activation in vascular smooth muscle. Am J Physiol Cell Physiol 289: C1343-C1350, 2005. 